\title{
Anatomía de la madera de Buddleja L. (Buddlejaceae): análisis fenético
}

\author{
Silvia Aguilar-Rodríguez $z^{1,2}$ \\ Teresa Terrazas ${ }^{1}$
}

\begin{abstract}
RESUMEN
El género Buddleja L. comprende cerca de 100 especies de distribución pantropical que varían desde pequeños arbustos hasta árboles de 20 a $30 \mathrm{~m}$ de alto y pocas son lianas. En este trabajo se presentan las características anatómicas de la madera de Buddleja con base en el estudio de 41 especies, de ellas se describen anatómicamente diez por primera vez y de 15 se sintetizan sus características de la literatura. Además, los caracteres de la madera de 41 especies del género se analizan a través de un análisis fenético. El género presenta porosidad de anular a difusa, placas de perforación simple, punteaduras intervasculares alternas y engrosamientos helicoidales en elementos de vaso y traqueidas vasculares; fibras libriformes, septadas en algunas especies; parénquima axial escaso o raro; radios heterogéneos tipo IIB y el $35 \%$ de las especies contienen cristales prismáticos de diferentes tamaños en las células de los radios. Los resultados del análisis fenético muestran que hay especies idénticas anatómicamente, sin embargo las especies del género forman dos grandes grupos que se distinguen con base en la combinación de caracteres cualitativos y cuantitativos, más otro conjunto de especies que se separan por la presencia de caracteres cualitativos. Finalmente se discuten algunas consideraciones taxonómicas sobre la posición de Buddleja en el orden Scrophulariales.
\end{abstract}

PALABRAS CLAVE:

Xilema secundario, porosidad anular, Buddleja, Loganiaceae, Scrophulariales.

\begin{abstract}
Buddleja L. comprises nearly 100 species with pantropical distribution, varying from small shrubs to trees 20 to 30 meters high and few lianas. In this study wood anatomical characteristics of Buddleja are presented based on the study of 41 species, of those 10 are described for the first time, and 15 synthesized from the literature. Also the wood features for 41 species of the genus were analized through a phenetic analysis. The genus showed ring to diffuse porosity, simple perforation plates, alternate intervascular pits, and helical thickenings in vessel elements and vascular tracheids; libriform fibers, with septa in some species; axial parenchyma scanty or rare; heterogeneous rays type IIB and $35 \%$ of the species contain prismatic crystals of different size in their ray cells. Phenetic analysis showed that several species are anatomically identical. However, most species conformed two large groups based on qualitative and quantitative wood features. Moreover, other species are distinctive based on the presence of unique qualitative characters. Finally, some taxonomic considerations on Buddleja position within the Scrophulariales order are discussed.
\end{abstract}

KEY WORDS:

Secondary xylem, ring porosity, Buddleja, Loganiaceae, Scrophulariales. 


\section{INTRODUCCIÓN}

Buddleja L. es un género reconocido por Linneo desde el siglo XVIII y ubicado en la tribu Buddleiae de la familia Scrophulariaceae por Bentham (1846). Solereder (1895) clasifica a Buddleja en la subfamilia Buddleioideae de la familia Loganiaceae; en esta subfamilia se incluyen géneros con hojas dentadas glandulares y tallo sin floema intraxilemático con corcho de origen en el periciclo. Posteriormente Wilhelm (1910) y Hutchinson (1973) separan a Buddleja de Loganiaceae por el tipo de polen y tricomas. Dahlgren (1975) ubica a Buddleja en la familia Buddlejaceae, pero en el orden Gentianales por contener iridoides. Cronquist (1981) reconoce a Buddlejaceae dentro de los Scrophulariales y más recientemente otros autores sugieren, con base en caracteres embriológicos y xilemáticos, remover a Buddlejaceae del orden Gentianales (Carlquist, 1992; Struwe y Albert, 1997) y los caracteres moleculares confirman su ubicación en el orden Scrophulariales (Oxelman et al., 1999).

Buddleja es un género de distribución pantropical, presente en el sur de Asia, Africa y América. Se reconocen alrededor de 100 especies (Mabberley, 1987), 50 se distribuyen en América, de las cuales 15 crecen en México (Norman, 1966). Leeuwenberg (1979) señala que existen alrededor de 16 en Africa y 21 en Asia. La mayoría son arbustos o árboles pequeños; sin embargo, algunas especies america-nas tienen entre 20 y $30 \mathrm{~m}$ de alto. Buddleja pulchella es la única especie trepadora del este y sureste de Africa. Se localizan desde los $0 \mathrm{msnm}$ al sur de Africa hasta los 4200 msnm en la región del Himalaya en Asia. Varias especies están relacionadas con los tipos de vegetación de las zonas montañosas en elevaciones de 2000 a $3500 \mathrm{~m}$ y otras se desarrollan en climas secos.

Los usos medicinales y alimenticios reportados para varias especies de Buddleja hacen evidente la importancia del género. Houghton (1984) menciona alrededor de 26 especies con algún uso en la medicina tradicional. A diversos órganos de $B$. cordata se les atribuyen propiedades analgésicas, eupépticas, diuréticas y antisépticas (Avendaño, 1996; Ordaz, 1996; Ortiz, 1996); las hojas de $B$. madagascarensis se empleaban en Madagascar como un sustituto de sopa y la planta se usa como remedio para asma y bronquitis. Otras especies se usan como veneno para peces (Standley, 1924). Hay pocos estudios sobre el uso de la madera del género, la de $B$. parviflora se emplea de manera local en la elaboración de postes o como leña de baja calidad (Aguilar et al., 2000).

Se han realizado varios estudios anatómicos de la madera de algunas especies de Budddleja que se resumen en Gregory (1994); así como las descripciones anatómicas para dos especies mexicanas por Quintanar et al. (1996) y Aguilar et al. (2000). Una interpretación sistemática a partir de las características anatómicas de la madera de Buddleja la presenta Mennega (1980) para 13 especies, 7 de América y 6 de Africa y Asia y el estudio de Carlquist (1997) para 23 especies de distribución principalmente americana.

\section{OBJETIVO}

Con el objetivo de ampliar el conocimiento de las características de la madera de Buddleja se estudió la anatomía de 26 especies, de ellas diez se describen anatómicamente por primera vez. Las especies estudiadas se comparan con las presentadas por Mennega (1980) y Carlquist (1997) y se analiza la similitud anatómica para 41 especies del género Buddleja.

\section{MATERIALES Y MÉTODO}

Se obtuvieron 50 muestras de madera pertenecientes a 26 especies de Buddleja de diferentes xilotecas y recolectas de 
campo (Tabla 1, Fig. 1). De las muestras procedentes de xiloteca, únicamente se usaron aquellas con madera madura para cuantificar los caracteres mensurables y las otras para corroborar su permanencia en el género. De las especies recolectadas en México se seleccionaron 2 ó 3 individuos maduros y sanos, dependiendo de su abundancia en cada localidad visitada. Se obtuvieron algunos datos generales de cada individuo, como hábito y altura; se acompañaron de localidad, tipo de vegetación y altitud. Para cada individuo seleccionado con hábito de árbol se obtuvo, a la altura del pecho una pequeña troza de madera (albura) y corteza de cinco $\mathrm{cm}$ de alto. La muestra de madera y corteza se fijó y almacenó en una solución de glicerinaalcohol etílico-agua (GAA, 1:2:3) hasta su procesamiento en el laboratorio. De los arbustos se obtuvo un cilindro de 10 a 15 $\mathrm{cm}$ de alto de la rama más gruesa, a una altura de cinco $\mathrm{cm}$ del suelo, la cual también se fijó en GAA. Se recolectó material de respaldo con flores o frutos del mismo individuo del que se tomó la muestra de madera. Este material se depositó en los herbarios del Colegio de Posgraduados (CHAPA) y del Campus Iztacala de la UNAM (IZTA).

Las muestras se cortaron de 20 ó 30 $\mu \mathrm{m}$ de grosor con un micrótomo de deslizamiento en los planos transversal, tangencial y radial; se tiñeron y montaron siguiendo el método convencional para madera (Johansen, 1940). Los disociados se prepararon con la solución de Jeffrey (Berlyn y Miksche, 1976) y sólo se hicieron preparaciones temporales para cuantificar la longitud de fibras y elementos de vaso, así como para corroborar la presencia de traqueidas vasculares y número de septos por fibra.

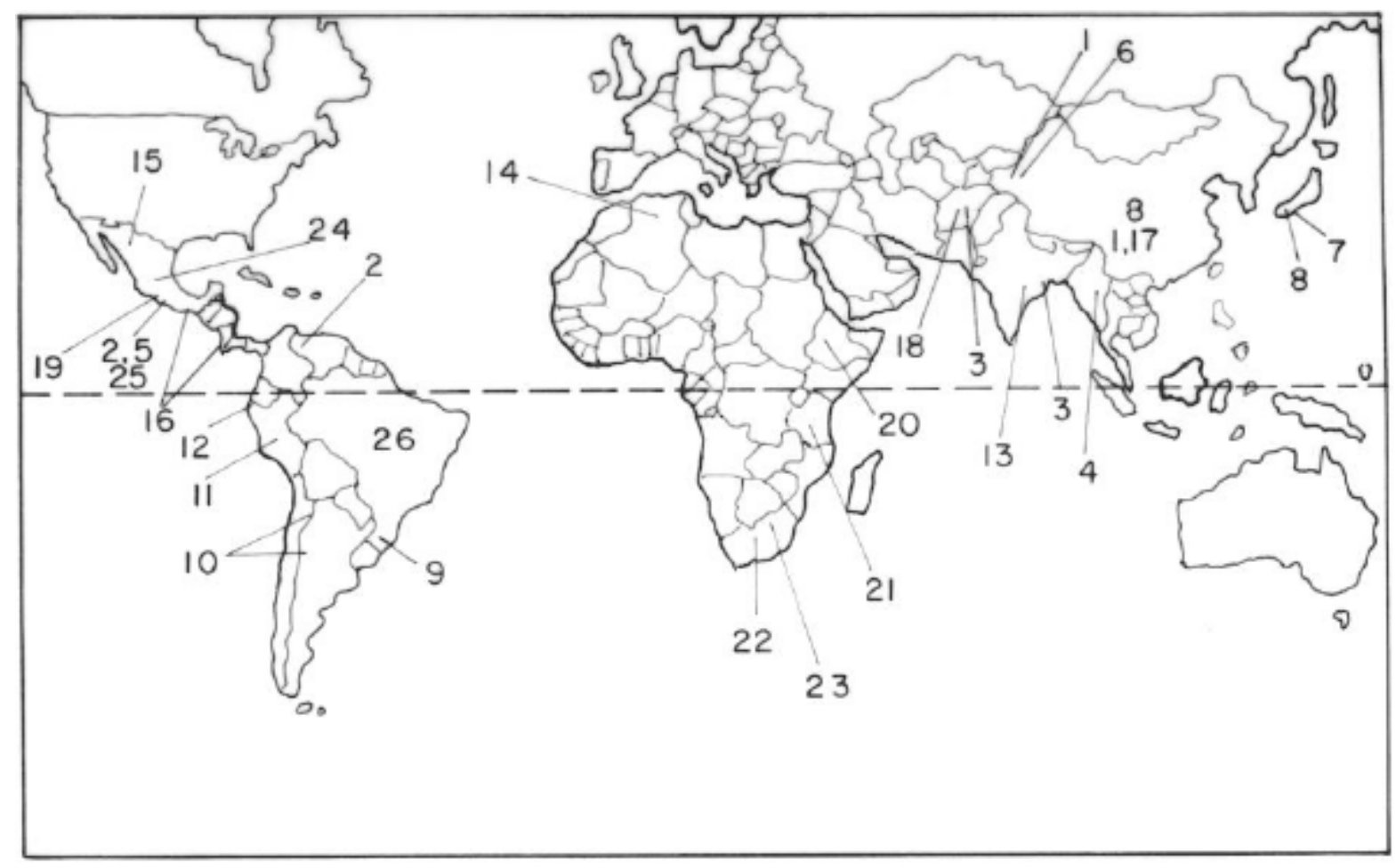

Figura 1. Mapa de distribución geográfica de las especies estudiadas de Buddleja. Los números se corresponden con los de la tabla 1 
Tabla 1. Número de xiloteca y procedencia de las especies de Buddleja estudiadas

(Los números en paréntesis corresponden a los presentados en la figura 1)

(1) B. alternifolia Maxim.: Uw840; FPRLw13170, China

(2) B. americana L.: CTFw23035, Venezuela; MEXU 876, México

(3) B. asiatica Lour.: Kw13213, India; BFHw13082, Birmania

(4) B. colvilei Hook. f. et Thoms.: Kw13216, Bengala

(5) B. cordata H.B.K.: Aguilar 72, 252, México

(6) B. crispa Benth.: Kw23025, China

(7) B. curviflora Hook. et Arn.: Tw14289; TWTw14276; TWTw15792, Japón

(8) B. davidii Franch.: RBRJw3.932; FPRLw12697; BFHw17007, China, Japón

(9) B. elegans Cham et Schlecht: Uw1372, Brasil

(10) B. globosa Hope: BFHw17597; Uw532; FPRLw21364; Kw23026, Perú, Argentina, Chile, en los Andes

(11) B. incana Ruíz et Pavón: Tw26409, Perú

(12) B. longifolia H.B.K.: BFHw10071, Perú

(13) B. macrostachya Benth.: Kw13218, India

(14) B. madagascariensis Lam.: Tw48506, Marruecos

(15) B. marrubifolia Benth.: Tejero 4035, México

(16) B. nitida Benth.: BFHw16102, BFHw19812, Costa Rica; Aguilar 284a, 284b, México

(17) B. nivea Duthie: Uw166; Uw167, China

(18) B. paniculata Wall.: Kw13219; FPRLw3033, Birmania

(19) B. parviflora H.B.K.: Aguilar 53, México; Kw13222, México; Kw13221, México; Uw15970, México; MEXU 934, México

(20) B. polystachya Fresen.: Kw13223, Etiopía

(21) B. pulchella N.E. Brown: Uw22028, Tanzania

(22) B. saligna Willd.: Uw902, cultivado; Kw13224, Sudáfrica; Uw22040, Sudáfrica

(23) B. salviifolia (L.) Lam.: Uw22041, Sudáfrica

(24) B. scordioides H.B.K.: Ávila s/n, México; Aguilar 270, 271, 273, México

(25) B. sessiliflora H.B.K.: Aguilar 71, 253, México

(26) B. thyrsoides Lam.: Uw20839, Brasil

Se cuantificaron y describieron los caracteres anatómicos siguiendo las recomendaciones de la Asociación Internacional de los Anatomistas de la Madera (IAWA Committee, 1989) y para radios las de Kribs (1935). Debido al dimorfismo observado en todas las especies se midió el diámetro del lumen de los vasos anchos y angostos y la longitud de los elementos de vaso anchos y angostos. También se midió el diámetro horizontal de punteaduras intervasculares; longitud, diámetro del lumen, diámetro total y grosor de pared de las fibras; así como altura y anchura de los radios multiseriados. Todos estos caracteres se cuantificaron con el analizador de imágenes Image-Pro Plus versión 3.1 (Media Cybernetics, 1997), adaptado a un microscopio Olympus BX-
50. Se tomaron 25 mediciones por carácter por muestra y se obtuvieron la media y la desviación estándar de cada carácter por especie.

La semejanza anatómica entre las especies de Buddleja se estimó mediante los métodos de clasificación (Sneath y Sokal, 1973) con el programa NTSYS-PC versión 1.80 (Rohlf, 1993). Se construyó una matriz básica de datos con 48 caracteres por 41 Unidades Taxonómicas Operativas (OTUs) (Apéndice I). Las OTUs correspondieron al mismo número de especies. La matriz de semejanza entre especies se obtuvo por el coeficiente de asociación de Dice-Sorensen y el método de agrupamiento se llevó a cabo por el promedio aritmético (UPGMA). 


\section{RESULTADOS}

\section{Descripción anatómica}

Anillos de crecimiento (Fig. 2-7). En la mayoría de las especies de Buddleja los anillos de crecimiento están marcados, reconociéndose cinco tipos. 1) En el $54 \%$ de las especies los anillos de crecimiento se delimitan por bandas de fibras de pared más gruesa con lúmenes pequeños y vasos con menor diámetro en la madera tardía, 2) en la madera tardía de Buddleja macrostachya sólo las fibras de lúmenes reducidos y paredes gruesas delimitan los anillos, 3) en $B$. asiatica y $B$. curviflora hay una disminución gradual en el diámetro de los vasos acompañada de una banda de fibras con lúmenes angostos, 4) en $B$. crispa el parénquima marginal, además de

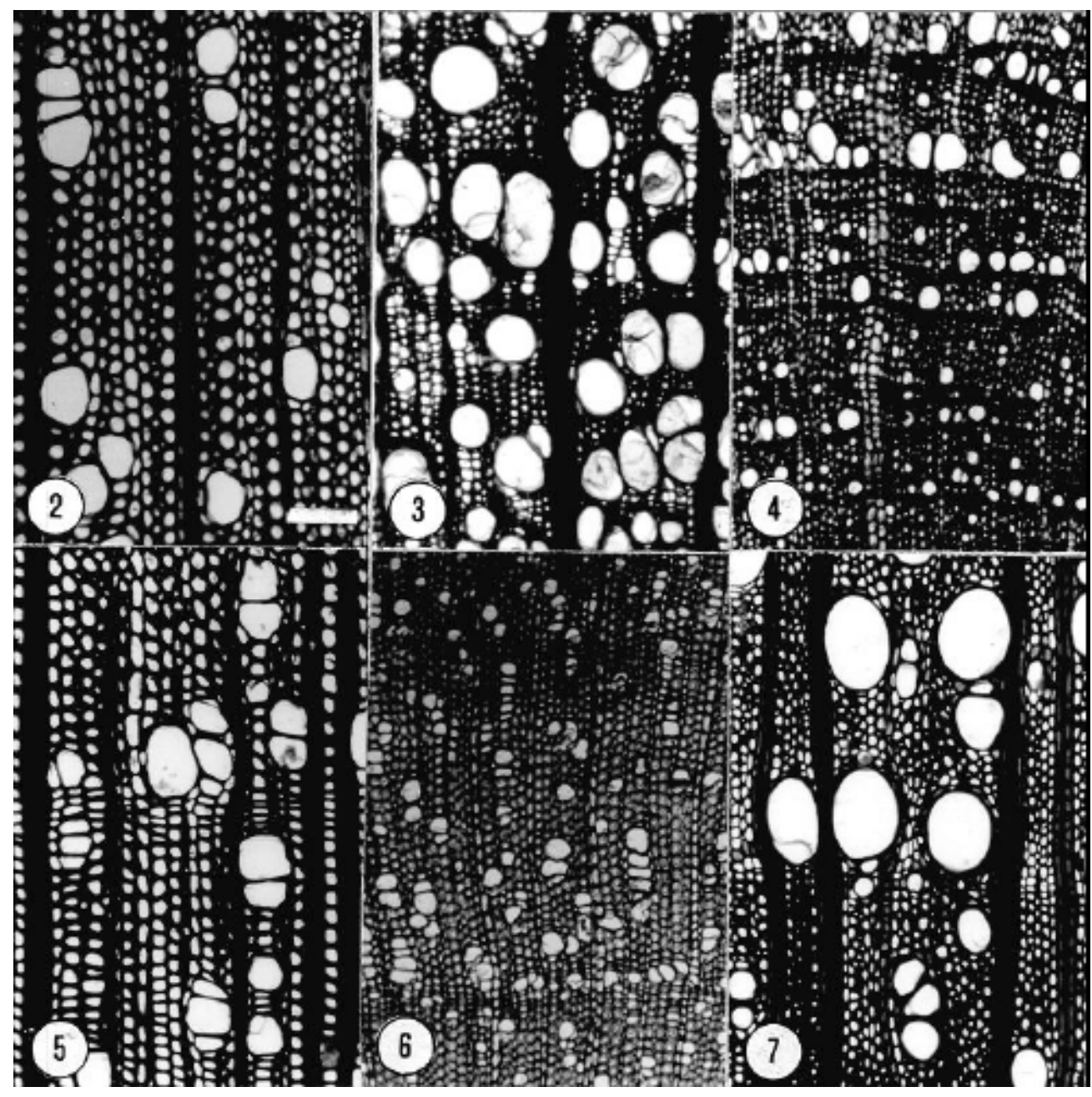

Figuras 2-7. Tipos de porosidad en el género Buddleja. Cortes transversales (Escala $=100 \mu \mathrm{m})$

2). B. americana, porosidad difusa 3). B. alternifolia, porosidad anular, tílides abundantes. 4). B. marrubifolia, porosidad anular. 5). B. macrostachya, porosidad difusa, los anillos de crecimiento delimitados sólo por las fibras de lúmenes reducidos y paredes gruesas. 6). B. elegans, porosidad anular. 7). B. asiatica, porosidad semianular, acompañada de una banda de fibras con lúmenes angostos. 
las paredes más gruesas de las fibras definen esta zona y 5) en $B$. longifolia el anillo de crecimiento se delimita en la madera tardía por la agrupación de vasos en cadenas radiales de 3 a 9 vasos. En individuos de $B$. cordata y $B$. parviflora los anillos son del tipo 10 no existen. En cuatro especies, $B$. nitida, $B$. americana, $B$. madagascariensis y $B$. thyrsoides, de porosidad difusa, no se observó diferencia entre la madera temprana y tardía. Las especies estudiadas sólo por Carlquist (1997) B. globosa y B. rufescens no presentan diferencias entre madera temprana y tardía y el resto presentó anillos de crecimiento del tipo 1 asignado en este estudio.

Vasos (Fig. 2-10). Los vasos son de contorno oval o anguloso vistos en corte transversal en cualquiera de los tipos de porosidad (anular, semianular y difusa). En las especies estudiadas, nueve presenta-ron una gradación entre porosidad semianular y difusa. Las especies con porosidad anular son $B$. davidii, B. colvilei, B. crispa, B. globosa, $B$. marrubifolia, $B$. nivea, $B$. scordioides y $B$. sessiliflora, con una marcada diferencia entre el diámetro de vasos de la madera temprana y la madera tardía. En las especies con porosidad anular, el diámetro de los vasos en la madera temprana tiene una media que varía de 29 $\mu \mathrm{m}$ en $B$. marrubifolia a $97 \mu \mathrm{m}$ en $B$. colvilei y en madera tardía de $20 \mu \mathrm{m}$ en $B$. marrubifolia a $33 \mu \mathrm{m}$ en $B$. nivea (Tabla 2 ). Se observa porosidad difusa en $B$. alternifolia, $B$. americana, $B$. incana, $B$. macrostachya, $B$. madagascariensis, $B$. nitida, B. polystachya, B. pulchella, $B$. saligna y $B$. thyrsoides; sin embargo, en estas especies también existe un dimorfismo marcado en el diámetro de los vasos. Los vasos anchos tienen una media que fluctúa de $35 \mu \mathrm{m}$ en $B$. saligna a $95 \mu \mathrm{m}$ en $B$. pulchella, mientras que en los vasos angostos varía de $17 \mu \mathrm{m}$ en $B$. thyrsoides a $52 \mu \mathrm{m}$ en $B$. nitida (Tabla 2). La longitud de los elementos de vaso anchos presenta una media de $178 \mu \mathrm{m}$ en $B$. marrubifolia a $616 \mu \mathrm{m}$ en $B$. macrostachya; en los vasos angostos varía de $208 \mu \mathrm{m}$ en $B$. marrubifolia a 600 $\mu \mathrm{m}$ en $B$. macrostachya (Tabla 2). La placa de perforación es simple con inclinación de 40-45; las punteaduras intervasculares son alternas con un diámetro que fluctúa de $5 \mu \mathrm{m}$ en $B$. polystachya a $10 \mu \mathrm{m}$ en $B$. americana, las punteaduras vaso-radio tienen bordes reducidos o son simples, excepto en $B$. madagascariensis, $B$. paniculata, $B$. pulchella y $B$. saligna, con punteaduras similares a las intervasculares.

Los elementos de vaso presentan engrosamientos helicoidales en todas las especies, excepto en Buddleja pulchella. Sin embargo, su presencia varía; por ejemplo, se observan engrosamientos en todos los vasos de $B$. alternifolia, $B$. cordata, $B$. curviflora, $B$. davidii, $B$. elegans, $B$. globosa, $B$. longifolia, $B$. macrostachya, $B$. marrubifolia, $B$. nitida, $B$. parviflora, $B$. saligna, $B$. salviifolia, $B$. scordioides, $B$. sessiliflora y $B$. thyrsoides; sólo en los vasos angostos de $B$. americana, $B$. colvilei y $B$. crispa; en los vasos angostos y en pocos vasos anchos de $B$. asiatica, $B$. incana, $B$. madagascarensis, $B$. nivea y $B$. paniculata y son poco evidentes en $B$. polystachya. Carlquist (1997) observó engrosamientos helicoidales en todos los vasos en $B$. araucana, $B$. cordobensis, $B$. mendozensis, $B$. racemosa y $B$. Tubiflora; solamente en las especies $B$. stachyoides, $B$. tucumanensis y $B$. utahensis encontró engrosamientos en los vasos angostos y en pocos anchos.

Se presentan traqueidas vasculares con engrosamientos helicoidales en $B$. colvilei, B. cordata, B. davidii, B. longifolia, $B$. nivea, $B$. parviflora, $B$. scordioides y $B$. Sessiliflora; así como en $B$. araucana, $B$. mendozensis, $B$. racemosa, $B$. tubiflora y B. utahensis (Carlquist, 1997). 


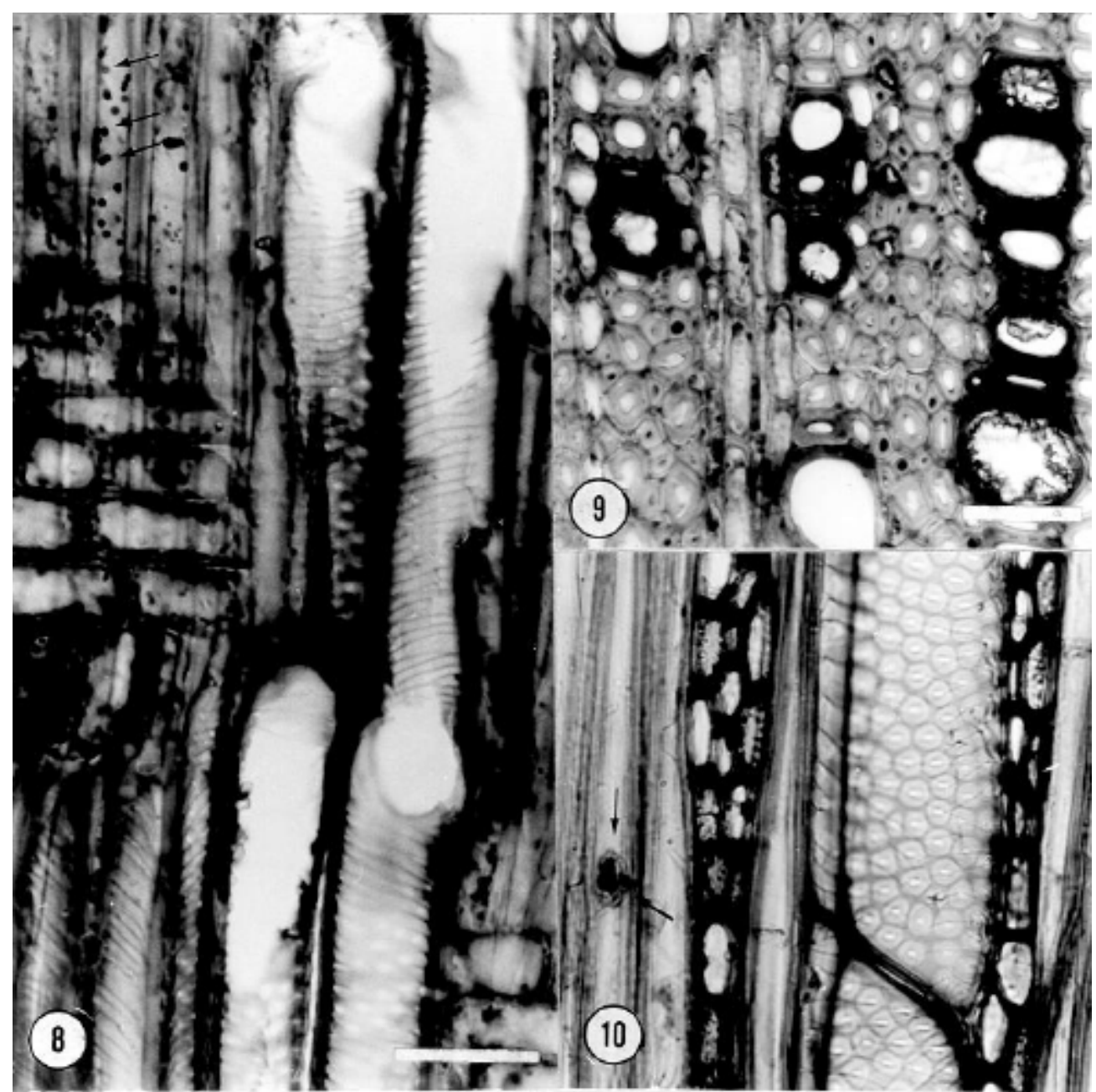

Figuras 8-10. Características de los elementos de vaso (Escala $=40 \mu \mathrm{m}$ ) 8). Corte tangencial de $B$. saligna, placa de perforación simple; engrosamientos helicoidales en vasos y contenidos en forma de gotas esféricas en fibras (flechas). 9). Corte transversal de $B$. saligna, cristales en el lumen de los vasos. 10). Corte tangencial de $B$. longifolia, punteaduras intervasculares alternas y traqueida vascular con engrosamientos helicoidales; fibra con núcleo y citoplasma contraído (flecha).

Las tílides ocluyen vasos grandes y pequeños de Buddleja alternifolia, $B$. colvilei, $B$. crispa, $B$. nitida y $B$. nivea; en $B$. pulchella la frecuencia de tílides es mayor en los vasos próximos a la médula. Buddleja saligna se distingue por la presencia de cristales con forma irregular en el lumen de algunos vasos.

Fibras (Fig. 8, 10-12). Las fibras son en su mayoría de tipo libriforme, aunque se observan abundantes fibrotraqueidas en $B$. marrubifolia y $B$. scordioides. Las fibras libriformes tienen punteaduras simples en 17 especies y punteaduras con areolas muy reducidas en $B$. alternifolia, $B$. colvilei, $B$. curviflora, $B$. incana, $B$. macrostachya, $B$. parviflora, $B$. polystachya, $B$. salviifolia y $B$. scordioides. Solamente nueve especies presentan fibras septadas $y$ en $B$. nivea se encuentran escasas fibras septadas. Carlquist (1997) también encontró fibras septadas en $B$. araucana, $B$. bullata, $B$. indica, $B$ skutchii, $B$. tubiflora y $B$. tucumanensis. La longitud promedio de 
las fibras fluctúa de $346 \mu \mathrm{m}$ en $B$. $\quad 23 \mu \mathrm{m}$ en $B$. macrostachya, con un grosor marrubifolia a $1148 \mu \mathrm{m}$ en $B$. promedio de la pared de $1.6 \mu \mathrm{m}$ en $B$. Macrostachya; el diámetro total de las scordioides a $4.1 \mu \mathrm{m}$ en B. colvilei (Tabla 2). fibras varía de $11 \mu \mathrm{m}$ en $B$. thyrsoides a

Tabla 2. Características cuantitativas de 26 especies de Buddleja (Valores de la media \pm una desviación estándar en $\mu \mathrm{m}$ )

\begin{tabular}{|c|c|c|c|c|c|c|}
\hline ESPECIES & DVA & DVAn & LVA & LVAn & DP & LFL \\
\hline B. alternifolia & $83 \pm 20$ & $24 \pm 8$ & $243 \pm 35$ & $258 \pm 38$ & $8 \pm 0.9$ & $587 \pm 102$ \\
\hline B. americana & $67 \pm 16$ & $39 \pm 17$ & $440 \pm 72$ & $368 \pm 88$ & $10 \pm 0.9$ & $1042 \pm 178$ \\
\hline B. asiática & $81 \pm 22$ & $33 \pm 8$ & $349 \pm 113$ & $387 \pm 109$ & $8 \pm 1.2$ & $867 \pm 111$ \\
\hline B. colvilei & $97 \pm 18$ & $27 \pm 5$ & $446 \pm 69$ & $493 \pm 82$ & $8 \pm 0.8$ & $703 \pm 94$ \\
\hline B. cordata & $42 \pm 8$ & $25 \pm 5$ & $338 \pm 70$ & $360 \pm 69$ & $8 \pm 0.7$ & $900 \pm 134$ \\
\hline B. crispa & $87 \pm 23$ & $26 \pm 5$ & $235 \pm 49$ & $235 \pm 40$ & $6 \pm 0.6$ & $457 \pm 100$ \\
\hline B. curviflora & $59 \pm 12$ & $22 \pm 6$ & $288 \pm 62$ & $320 \pm 76$ & $7 \pm 0.6$ & $580 \pm 109$ \\
\hline B. davidii & $66 \pm 17$ & $30 \pm 6$ & $328 \pm 70$ & $358 \pm 63$ & $8 \pm 1.3$ & $703 \pm 108$ \\
\hline B. elegans & $24 \pm 6$ & $16 \pm 3$ & $302 \pm 70$ & $352 \pm 69$ & $7 \pm 0.9$ & $496 \pm 102$ \\
\hline B. globosa & $49 \pm 14$ & $26 \pm 6$ & $281 \pm 58$ & $295 \pm 54$ & $8 \pm 0.7$ & $612 \pm 81$ \\
\hline B. incana & $41 \pm 9$ & $21 \pm 4$ & $366 \pm 82$ & $350 \pm 66$ & $9 \pm 0.8$ & $761 \pm 129$ \\
\hline B. longifolia & $69 \pm 17$ & $37 \pm 5$ & $376 \pm 46$ & $387 \pm 50$ & $9 \pm 0.6$ & $933 \pm 124$ \\
\hline B. macrostachya & $73 \pm 12$ & $42 \pm 6$ & $616 \pm 155$ & $600 \pm 116$ & $9 \pm 0.6$ & $1148 \pm 143$ \\
\hline B. madagascariensis & $84 \pm 17$ & $40 \pm 9$ & $341 \pm 124$ & $414 \pm 65$ & $7 \pm 0.4$ & $776 \pm 107$ \\
\hline B. marrubifolia & $29 \pm 6$ & $20 \pm 5$ & $178 \pm 43$ & $208 \pm 36$ & $6 \pm 0.8$ & $346 \pm 53$ \\
\hline B. nitida & $94 \pm 44$ & $52 \pm 22$ & $411 \pm 89$ & $426 \pm 90$ & $9 \pm 1.6$ & $987 \pm 183$ \\
\hline B. nivea & $54 \pm 13$ & $33 \pm 8$ & $449 \pm 83$ & $463 \pm 88$ & $6 \pm 0.8$ & $914 \pm 139$ \\
\hline B. paniculata & $57 \pm 20$ & $34 \pm 7$ & $428 \pm 79$ & $435 \pm 65$ & $7 \pm 0.5$ & $867 \pm 124$ \\
\hline B. parviflora & $69 \pm 17$ & $34 \pm 7$ & $373 \pm 81$ & $353 \pm 55$ & $8 \pm 0.8$ & $898 \pm 174$ \\
\hline B. polystachya & $44 \pm 13$ & $24 \pm 8$ & $304 \pm 111$ & $301 \pm 54$ & $5 \pm 0.3$ & $641 \pm 126$ \\
\hline B. pulchella & $95 \pm 10$ & $41 \pm 10$ & $533 \pm 56$ & $529 \pm 69$ & $9 \pm 0.4$ & $771 \pm 98$ \\
\hline B. saligna & $35 \pm 7$ & $18 \pm 4$ & $266 \pm 42$ & $291 \pm 44$ & $7 \pm 1.6$ & $634 \pm 105$ \\
\hline B. salviifolia & $49 \pm 13$ & $32 \pm 5$ & $347 \pm 48$ & $362 \pm 69$ & $7 \pm 0.3$ & $693 \pm 68$ \\
\hline B. scordioides & $62 \pm 7$ & $24 \pm 6$ & $180 \pm 36$ & $211 \pm 37$ & $6 \pm 0.4$ & $424 \pm 92$ \\
\hline B. sessiliflora & $57 \pm 11$ & $32 \pm 8$ & $210 \pm 65$ & $304 \pm 79$ & $7 \pm 0.8$ & $542 \pm 90$ \\
\hline B. thyrsoides & $36 \pm 8$ & $17 \pm 3$ & $267 \pm 52$ & $272 \pm 38$ & $6 \pm 0.5$ & $556 \pm 74$ \\
\hline $\begin{array}{l}\text { DVA }=\text { diámetro del lu } \\
\text { DVAn }=\text { diámetro del lu } \\
\text { LVA }=\text { longitud de ele }\end{array}$ & 1. & sto & $\begin{array}{l}\text { LVAn = } \\
\text { DP }= \\
\text { LFL }=\end{array}$ & - & & $\begin{array}{l}\text { angosto } \\
\text { ascular }\end{array}$ \\
\hline
\end{tabular}


Tabla 2. Características cuantitativas de 26 especies de Buddleja (cont.) (Valores de la media \pm una desviación estándar en $\mu \mathrm{m}$ )

\begin{tabular}{|c|c|c|c|c|c|}
\hline ESPECIES & DTFL & DLFL & GPF & ARM & AnRM \\
\hline B. alternifolia & $13 \pm 1$ & $7 \pm 1.2$ & $2.6 \pm 0.4$ & $284 \pm 96$ & $39 \pm 9$ \\
\hline B. americana & $20 \pm 4$ & $13 \pm 3.0$ & $3.3 \pm 1.2$ & $331 \pm 159$ & $31 \pm 5$ \\
\hline B. asiática & $15 \pm 4$ & $9 \pm 2.6$ & $3.3 \pm 0.9$ & $448 \pm 132$ & $45 \pm 10$ \\
\hline B. colvilei & $20 \pm 3$ & $12 \pm 2.8$ & $4.1 \pm 0.8$ & $919 \pm 287$ & $69 \pm 8$ \\
\hline B. cordata & $14 \pm 2$ & $9 \pm 2.4$ & $2.4 \pm 0.4$ & $347 \pm 89$ & $32 \pm 6$ \\
\hline B. crispa & $13 \pm 2$ & $8 \pm 1.5$ & $2.4 \pm 0.3$ & $415 \pm 189$ & $23 \pm 3$ \\
\hline B. curviflora & $13 \pm 2$ & $6 \pm 1.7$ & $3.2 \pm 0.6$ & $345 \pm 128$ & $36 \pm 6$ \\
\hline B. davidii & $15 \pm 2$ & $9 \pm 1.9$ & $3.1 \pm 0.7$ & $690 \pm 352$ & $41 \pm 9$ \\
\hline B. elegans & $12 \pm 1$ & $6 \pm 1.2$ & $3.0 \pm 0.6$ & $576 \pm 167$ & $25 \pm 4$ \\
\hline B. globosa & $13 \pm 2$ & $8 \pm 2.5$ & $2.5 \pm 0.7$ & $373 \pm 150$ & $33 \pm 5$ \\
\hline B. incana & $14 \pm 2$ & $7 \pm 1.4$ & $3.4 \pm 0.5$ & $268 \pm 66$ & $40 \pm 6$ \\
\hline B. longifolia & $17 \pm 2$ & $10 \pm 2.5$ & $3.1 \pm 0.4$ & $389 \pm 50$ & $24 \pm 4$ \\
\hline B. macrostachya & $23 \pm 3$ & $16 \pm 3.0$ & $3.5 \pm 0.6$ & $640 \pm 241$ & $53 \pm 13$ \\
\hline B. madagascariensis & $16 \pm 2$ & $9 \pm 2.0$ & $3.4 \pm 0.5$ & $599 \pm 206$ & $33 \pm 6$ \\
\hline B. marrubifolia & $13 \pm 2$ & $6 \pm 1.3$ & $3.2 \pm 0.5$ & $456 \pm 120$ & $50 \pm 10$ \\
\hline B. nitida & $19 \pm 3$ & $12 \pm 3.0$ & $3.4 \pm 0.8$ & $432 \pm 145$ & $38 \pm 8$ \\
\hline B. nivea & $16 \pm 3$ & $10 \pm 2.2$ & $2.7 \pm 0.4$ & $457 \pm 149$ & $36 \pm 7$ \\
\hline B. paniculata & $17 \pm 2$ & $11 \pm 2.4$ & $3.2 \pm 0.4$ & $521 \pm 206$ & $45 \pm 7$ \\
\hline B. parviflora & $19 \pm 3$ & $12 \pm 3.0$ & $3.3 \pm 0.6$ & $335 \pm 122$ & $35 \pm 6$ \\
\hline B. polystachya & $16 \pm 3$ & $9 \pm 2.0$ & $3.3 \pm 0.5$ & $421 \pm 138$ & $36 \pm 5$ \\
\hline B. pulchella & $15 \pm 2$ & $8 \pm 2.0$ & $3.8 \pm 0.6$ & $677 \pm 257$ & $31 \pm 5$ \\
\hline B. saligna & $14 \pm 2$ & $7 \pm 1.5$ & $3.5 \pm 0.9$ & $328 \pm 130$ & $30 \pm 6$ \\
\hline B. salviifolia & $15 \pm 2.0$ & $9 \pm 2$ & $3.1 \pm 0.3$ & $501 \pm 183$ & $36 \pm 9$ \\
\hline B. scordioides & $16 \pm 3.0$ & $13 \pm 3$ & $1.6 \pm 0.2$ & $446 \pm 162$ & $65 \pm 14$ \\
\hline B.sessiliflora & $13 \pm 2$ & $7 \pm 1.0$ & $3.0 \pm 0.5$ & $465 \pm 178$ & $32 \pm 8$ \\
\hline B. thyrsoides & $11 \pm 2$ & $6 \pm 1.0$ & $2.6 \pm 0.4$ & $234 \pm 70$ & $20 \pm 3$ \\
\hline
\end{tabular}


Los contenidos en el lumen celular de las fibras son escasos. En las fibras de $B$. crispa, $B$. saligna y $B$. sessiliflora se presentan gotas esféricas de naturaleza fenólica. Buddleja madagascariensis y $B$. nivea presentan residuos de citoplasma en el lumen celular y se observaron núcleos, exclusivamente, en $B$. colvilei, $B$. longifolia y $B$. thyrsoides. Residuos de citoplasma han sido descritos también en B. skutchii y B. colvilei (Carlquist, 1997).

Parénquima axial (Fig. 2-7). El parénquima axial está ausente en $B$. elegans, $B$. longifolia y $B$. marrubifolia, al igual que en $B$. australis, $B$. indica y $B$. mendozensis (Carlquist, 1997; Mennega, 1980). La mayoría de las especies presentan parénquima paratraqueal escaso; ocasionalmente, se observa vasicéntrico en $B$. asiatica, $B$. crispa y $B$. nitida. El parénquima apotraqueal difuso se observa ocasionalmente en $B$. alternifolia, $B$. americana, $B$. asiatica, $B$. crispa, $B$. paniculata, $B$. parviflora y $B$. scordioides. En $B$. crispa hay bandas angostas marginales y Carlquist (1997) encuentra bandas de parénquima inicial en $B$. salviifolia. Los contenidos en el lumen de las células del parénquima axial son escasos; únicamente $B$. colvilei y $B$. paniculata presentan gotas esféricas de naturaleza fenólica.

Parénquima radial (Fig. 11-15). Los radios son del tipo heterogéneo IIB (Kribs, 1935). Los uniseriados se presentan escasos en la mayoría de las especies. Los multiseriados tienen una altura promedio de $234 \mu \mathrm{m}$ en $B$. thyrsoides a $919 \mu \mathrm{m}$ B. colvilei (Tabla 2) y comúnmente de 2 a 4 células de ancho, pero en $B$. asiatica, $B$. davidii y $B$. paniculata se presentan radios de 3 a 6 células de ancho. Los radios biseriados más angostos corresponden a $B$. thyrsoides con $20 \mu \mathrm{m}$ de ancho y los 3 a 4 seriados más anchos, a $B$. colvilei con $69 \mu \mathrm{m}$ (Tabla 2). En B. madagascariensis, $B$. pulchella y $B$. davidii (BFH-17007) los radios se unen en sus márgenes uniseriados. En las células de los radios los contenidos son más abundantes. Se observan gotas esféricas de color obscuro de naturaleza fenólica en $B$. asiatica, $B$. colvilei, B. crispa, B. macrostachya, $B$. nitida, $B$. paniculata y $B$. saligna. El $35 \%$ de las especies estudiadas presentan cristales que varían en tamaño y abundancia. Hay cristales prismáticos grandes $(>16 \mu \mathrm{m})$ y abundantes en $B$. globosa, B. marrubifolia y $B$. scordioides; son medianos (10 a $15 \mu \mathrm{m}$ ) en $B$. cordata (Aguilar 72), B. incana, B. parviflora y $B$. thyrsoides; se presentan pequeños $(<6$ $\mu \mathrm{m}$ ) y escasos en B. cordata (Aguilar 252), $B$. saligna y $B$. sessiliflora; y en algunas ocasiones se observan como pequeñas aglomera-ciones, pero nunca cristales del tipo estiloide. Carlquist (1997) también señala cristales grandes en $B$. cordobensis, $B$. mendozensis y $B$. utahensis y pequeños en $B$. tubiflora.

\section{Análisis fenético}

El fenograma (Fig. 16) basado en el coeficiente de asociación de DiceSorensen (Apéndice II) revela la existencia de dos grupos además de $B$. parviflora y $B$. scordioides, $B$. colvilei, $B$. macrostachya y $B$. crispa en una posición externa a ellos. Los dos grupos se relacionan a una distancia de $0.81 ; B$. parviflora y $B$. scordioides se encuentran unidos a estos dos grupos a una distancia de 0.76 , mientras que $B$. colvilei y $B$. macrostachya lo hacen a una distancia de $0.73, B$. crispa se encuentra unida a estos a una distancia de 0.72. El grupo I se define a una distancia de 0.82 e incluye a 12 especies, separándose en este nivel $B$. saligna del resto del grupo, también $B$. thyrsoides se separa del grupo a una distancia de $0.83 ; B$. curviflora y $B$. salviifolia son las especies más similares anatómicamente (0.98). El grupo II se define a una distancia de 0.83 e incluye 24 especies que se separan en dos subgrupos, el IIA se encuentra a una distancia de 0.84, separándose en este nivel 13 especies, siendo $B$. nivea y $B$. paniculata las más diferentes anatómicamente del grupo (0.84) y $B$. americana, $B$. rufescens y $B$. stachyoides las más parecidas anatómicamente, a una 
distancia de 0.97; B. madagascariensis, $B$. bullata y $B$. skutchii tienen el valor máximo de asociación (1.0) y se consideran idénticas en sus características anatómicas. En el subgrupo IIB se asocian 11 especies a una distancia de 0.89 , aquí $B$. marrubifolia y $B$. mendozensis se separan de $B$. elegans, $B$. anchoensis, $B$. domingensis, B. cordobensis, B.australis, $B$. longifolia, $B$. racemosa, $B$. araucana y $B$. tubiflora. Las especies $B$. elegans, $B$. anchoensis y $B$. domingensis y $B$. longifolia y $B$. racemosa tienen el valor máximo de asociación de 1.0.

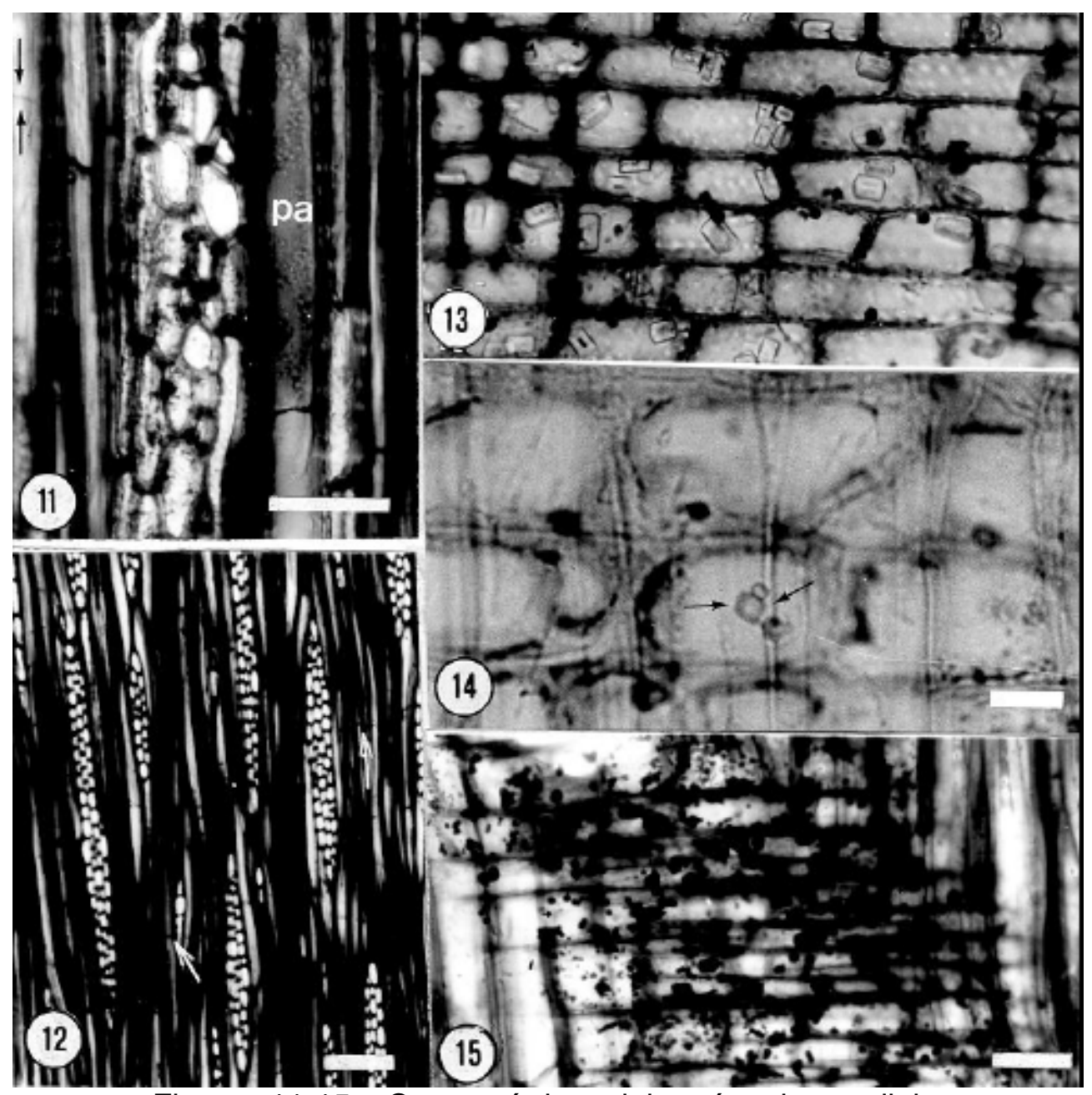

Figuras 11-15. Características del parénquima radial

(Escalas: 11 y $13=40 \mu \mathrm{m} ; 12=100 \mu \mathrm{m} ; 14=10 \mu \mathrm{m} ; 15=50 \mu \mathrm{m}$ )

11-12. Corte tangencial. 11). B. madagascariensis, radio multiseriado, serie parenquimatosa (pa) y fibras septadas (flecha). 12). $B$. nitida, radios uni y triseriados, fibras septadas (flecha). 13-15. Corte radial. 13). B. parviflora, cristales prismáticos medianos. 14). B. saligna, cristales prismáticos pequeños (flecha). 15). B. nitida, contenidos en forma de gotas esféricas. 


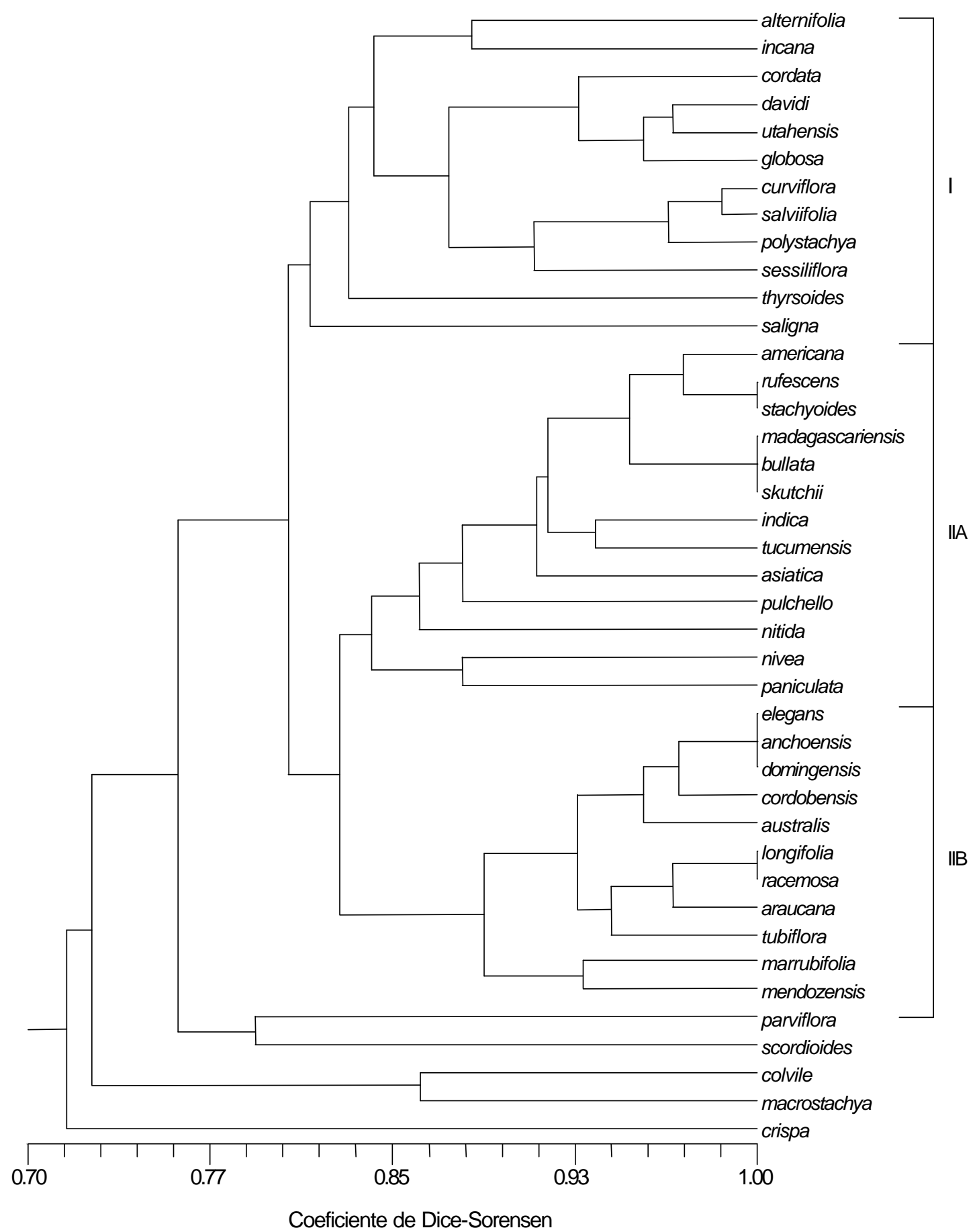

Figura 16. Fenograma por el método de agrupamiento (UPGMA) para 41 especies del género Buddleja 


\section{DISCUSIÓN}

El género Buddleja se caracteriza por presentar anillos de crecimiento delimitados por una diferenciación gradual en el diámetro de los vasos o por fibras y, en pocas ocasiones, por parénquima axial. Sin embargo, este carácter parece estar más asociado con aspectos ambientales y fisiológicos que con aspectos taxonómicos (Metcalfe y Chalk, 1983, Carlquist, 1988). Los elementos celulares que se forman cuando existe mayor humedad tienen paredes relativamente delgadas y un lumen grande, pero cuando el suministro de agua es restringido se producen células con paredes más gruesas y un lumen más pequeño. Estas características se observan principalmente en especies que crecen en zonas donde hay estacionalidad ambiental. Buddleja es un género relacionado con tipos de vegetación que prosperan en las zonas montañosas, en donde hay restricción de agua durante el invierno, así como en zonas de climas secos, en donde existen periodos de sequía, condiciones que se manifiestan en la madera de algunas especies del género.

Por otro lado, fue difícil definir el tipo de porosidad en varias especies, debido al continuum de variación en el diámetro de los vasos a través de los anillos. Sólo se observó porosidad anular evidente en $B$. colvilei, B. crispa, B. davidii, B. globosa, B. marrubifolia, $B$. nivea, $B$. sessiliflora y $B$. scordioides y parece estar asociada al ambiente donde se desarrollan, que son matorrales de zonas secas o templadas (Fig. 1). Carlquist (1997) menciona que el término porosidad anular se estableció para maderas de zonas templadas del norte, donde los cambios estacionales drásticos, sobre todo en la temperatura, coinciden con la cantidad de agua disponible. La presencia de porosidad anular tiene que ver más con heladas que con cambios graduales de temperatura a lo largo de un año y a pesar de que varias especies de Buddleja son típicas de zonas elevadas, es un género de distribución pantropical principalmente, con algunos representantes que alcanzan latitudes no mayores a los $50^{\circ}$.

Los engrosamientos helicoidales están presentes en todas las especies de Buddleja, excepto en B. pulchella. Estos engrosamientos pueden ser usados como un buen carácter taxonómico; sin embargo, su presencia, así como sus características también se pueden asociar a condiciones ecológicas (Metcalfe y Chalk, 1983). Los engrosamientos helicoidales están relacionados con el estrés hídrico (Carlquist, 1988). En floras extremadamente áridas la incidencia de engrosamientos helicoidales es muy baja o moderadamente alta, siendo muy alta en zonas mediterráneas 0 vegetación esclerófila (Baas, 1983). Carlquist (1982) propone que los engrosamientos helicoidales tienen la finalidad de incrementar la superficie de la pared del vaso aumentando la cantidad de agua que puede adherirse a la superficie; además evita la formación de embolismos cuando existe estrés hídrico. Las especies de Buddleja crecen en sitios expuestos a fluctuaciones estacionales, ya sea por frío o por sequía, por lo que no es rara la presencia de estos engrosamientos en las paredes de sus vasos. La frecuencia de los engrosamientos helicoidales en los vasos angostos y anchos podría explicarse en términos del grado y duración de los periodos de sequía a la que estarían expuestas las diferentes especies en sus respectivos ambientes.

Las tílides pueden ser consideradas como un carácter ambiguo y su presencia o ausencia en la mayoría de los casos no son un carácter taxonómico (Carlquist, 1988); sin embargo, Chalk (1983) considera que debido a su esporádica y restringida presencia son de valor diagnóstico en algunas familias, por lo que también pueden ser usadas con cautela en los estudios filogenéticos (Herendeen y Miller, 2000) y éste sería el caso de Buddleja.

Las fibras de tipo libriforme son comunes en el género Buddleja, pero en 
nueve especies se observaron fibras con areolas muy reducidas. Mennega (1980) describió tanto punteaduras simples como punteaduras angostamente areoladas en la pared radial y en menor número sobre la pared tangencial, similares a las encontradas en este estudio. La morfología de las punteaduras en las fibras de angiospermas es un tema que todavía se discute. Según Baas (1999) el término punteadura areolada debe seguir empleándose, debido a que tiene connotación evolutiva. Para Magendans (1999) punteadura areolada está asociada con punteadura caliciforme, que difiere de la areolada por un ensanchamiento gradual más que abrupto del canal de la punteadura hacia la lámina media. Mientras continúe esta controversia, se sugiere seguir lo recomendado por la Asociación Internacional de Anatomistas de la Madera (IAWA Committee, 1989).

El $35 \%$ de las especies presentan fibras septadas. Carlquist (1997) señala que los septos son indicadores de longevidad en las fibras, por lo que éstas pueden ser llamadas también fibras vivas. Esta característica se relaciona con maderas donde el parénquima es escaso (Harrar, 1946) y se incrementa conforme el parénquima decrece (Spackman y Swamy, 1948), por lo que las fibras septadas se consideran como un tipo de células de almacenamiento parecidas al parénquima axial (Carlquist, 1988). Si esto es cierto, se debería esperar una mayor incidencia de especies con fibras septadas debido a la escasez del parénquima axial que caracteriza al género Buddleja. Sin embargo, en las fibras no septadas de $B$. colvilei, $B$. longifolia, $B$. madagascariensis, $B$. nivea y $B$. thyrsoides se observaron restos de citoplasma o núcleos, Carlquist (1997) los encontró también en las fibras de $B$. americana, $B$. colvilei y $B$. skutchii y Wolkinger (1970) en las de $B$. alternifolia y $B$. davidii. En maderas con parénquima axial escaso también son frecuentes las fibras nucleadas (Metcalfe y Chalk, 1983), así como en algunas formas escandentes, subarbustivas y arbustivas (Rajput y Rao, 1999). Las fibras no septadas pueden mantener protoplasto y núcleo vivos con la finalidad de almacenar material de reserva y ser usado en el momento de reiniciar la actividad cambial. Posiblemente el número de especies con fibras no septadas vivas aumentaría en Buddleja si el material se preservara en algún líquido después de ser colectado.

El tipo de radios que caracteriza al género Buddleja es heterogéneo IIB (Kribs 1935). Carlquist (1997) hace referencia a radios paedomórficos tipo I en Buddleja americana, $B$. colvilei y $B$. scordioides, lo cual contrasta con los radios encontrados para estas especies. Buddleja americana y $B$. colvilei son arbustos o árboles de hasta 6 ó $10 \mathrm{~m}$ de alto con diámetros de 20 $\mathrm{cm}$ y Buddleja scordioides es un arbusto no mayor a $1 \mathrm{~m}$ de alto con diámetro de 2 $\mathrm{cm}$; las tres especies presentan los radios heterogéneos típicos del género. Los radios, además del transporte radial de nutrientes almacenan sustancias nutritivas y compuestos ergásticos (Metcalfe y Chalk, 1983).

Los cristales son de las sustancias ergásticas más comunes en angiospermas, su presencia está asociada con varias funciones de la planta o con la edad del individuo, así como con el clima y tipo de suelo. Los cristales se consideran sustancias dinámicas que llegan a transformarse; sin embargo, el tipo y distribución pueden ser lo suficientemente estables para tomarse en cuenta con propósitos diagnósticos (Metcalfe y Chalk, 1983). En Buddleja la presencia y tamaño de los cristales se interpreta como un carácter diagnóstico en nueve especies del género. Por ejemplo, cristales prismáticos mayores de $16 \mu \mathrm{m}$ distinguen a $B$. globosa, $B$. marrubifolia y $B$. scordioides y cristales menores a $6 \mu \mathrm{m}$ a $B$. saligna y $B$. sessiliflora. Buddleja cordata fue la única especie con cristales de dos tamaños en diferentes individuos, por lo que se sugiere un muestreo más amplio que permita entender su variación. 


\section{Análisis fenético}

Los grupos revelados en el fenograma tienen muy pocos caracteres únicos que los distinguen; sin embargo, caracteres cuantitativos y cualitativos permiten reconocer grupos de especies. Por ejemplo, en el grupo I se asocian las especies que presentan vasos anchos con diámetro mediano (34 a $66 \mu \mathrm{m}$ ), fibras con punteaduras semiareoladas, con diámetro de la fibra pequeño (8 a $15 \mu \mathrm{m}$ ) y longitud mediana (385 a $767 \mu \mathrm{m}$ ). La asociación de $B$. saligna dentro del grupo I se distingue por la presencia de cristales irregulares en los vasos, carácter exclusivo para esta especie. Mientras que la posición externa de $B$. thyrsoides dentro de este grupo se debe a la presencia de diámetros de vaso angostos de $17 \mu \mathrm{m}$ y radios con una anchura de $20 \mu \mathrm{m}$. Buddleja curviflora y $B$. salviifolia son especies que sólo se diferencian entre sí por la presencia de fibras septadas en $B$. curviflora.

El grupo II incluye 24 especies que se separan en dos subgrupos. En el subgrupo IIA se asocian 13 especies por la presencia de engrosamientos helicoidales, la ausencia de anillos de crecimiento evidentes en la mayoría de las especies y fibras más largas (768 a 1150 $\mu \mathrm{m})$. Buddleja madagascariensis, $B$. bullata y $B$. skutchii tienen características idénticas al presentar el valor máximo de 1.0 , pero se diferencian del resto, por el tamaño de los elementos de vaso ancho y angostos $(<400 \mu \mathrm{m})$, el diámetro de las punteaduras intervasculares $(<7 \mu \mathrm{m})$ y el grosor de la pared de la fibra $(>3.4 \mu \mathrm{m})$. Buddleja americana, $B$. rufescens y $B$. stachyoides son muy similares anatómicamente, distinguiéndose $B$. americana de las otras dos, por la presencia de parénquima apotraqueal difuso. En el subgrupo IIB se asocian 11 especies con base principalmente en las punteaduras simples en fibras y el parénquima axial raro o escaso. Buddleja marrubifolia y $B$. mendozensis se separan de las demás debido a la presencia de cristales prismáticos grandes en sus radios; estos cristales están ausentes en el resto de las especies del grupo ( $B$. elegans, $B$. anchoensis y $B$. domingensis y $B$. longifolia, $B$. racemosa tienen un valor de asociación de 1.0), excepto en $B$. cordobensis, que también los presenta grandes $y$ además tiene traqueidas vasculares.

Buddleja crispa se distingue por el parénquima marginal, carácter que no comparte con ninguna de las especies estudiadas. Buddleja colvilei y $B$. macrostachya se separan de los dos grupos por la presencia de radios altos y anchos, aunque tienen de 2 a 4 células de grosor y $B$. parviflora y $B$. scordioides por ser las únicas especies con parénquima apotraqueal difuso y paratraqueal escaso.

Los resultados de este análisis indican que las especies de Buddleja son muy similares anatómicamente. Sin embargo, los estados de carácter para algunos caracteres cuantitativos en conjunto con los cualitativos permiten reconocer grupos de especies. Resultados similares se han encontrado en el género Tapirira (Terrazas y Wendt, 1995).

\section{Comentarios sobre sistemática}

Buddleja es el género más numeroso de los ocho reconocidos en Buddlejaceae (Brummitt, 1992). La posición taxonómica de Buddlejaceae ha sido inestable (Struwe y Albert, 1997), sin embargo análisis cladísticos a nivel suprafamiliar basados en caracteres morfológicos, anatómicos, fitoquímicos y moleculares, reconocen la posición de Buddlejaceae en el orden Scrophulariales (Bremer et al., 1998; Jensen, 1992; Oxelman, et al., 1999). La ausencia de floema intraxilemático, vasos de diámetro pequeño, engrosamientos helicoidales, punteaduras intervasculares pequeñas, así como distribución muy esparcida o ausente del parénquima axial son caracteres del xilema secundario que Buddlejaceae comparte con algunos miembros de Scrophulariales (Carlquist, 1992; Cronquist, 1981; Metcalfe y Chalk, 1950). 
Las relaciones entre los géneros (Androya, Buddleja, Emorya, Gomphostigma, Nuxia, Peltanthera, Polypremum y Sanango) de Buddlejaceae no son claras. Con base en caracteres anatómicos de la madera, Mennega (1980) menciona que Androya presenta una combinación de caracteres que sugieren una alianza con Oleaceae, mientras que Hunziker y $\mathrm{Di}$ Fulvio (1958) analizan características macromorfológicas de Peltanthera sugiriendo que debe ser incluido en Loganiaceae y Dickison (1994) excluye a Sanango de Buddlejaceae por la presencia de placas de perforación escalariforme y propone su ubicación en Gesneriaceae. Struwe y Albert (1997) ubican a Sanango en Gesneriaceae (Scrophulariales) como lo propone Dickison (1994) y a Polypremum en Scrophulariaceae, dejando en Buddlejaceae sólo a Androya, Buddleja, Emorya, Gomphostigma, Nuxia y Peltanhera. Evidencias basadas en análisis combinado de secuencias de $n d h \mathrm{~F}$ y $r b c \mathrm{~L}$ revelaron que solamente Buddleja, Emorya, Gom-phostigma y Nicodemia forman parte de la familia Buddlejaceae (Oxelman et al., 1999). Bisset et al. (1980) y Leeuwenberg y Leenhouts (1980) sugieren que Emorya y Nicodemia deben ser incluidas en Buddleja. Esta consideración es apoyada con base en la similitud de la madera de E. suaveolens con las especies de Buddleja distribuidas en zonas áridas (Carlquist, 1997). Sin embargo, Oxelman et al. (1999) señalan que esta propuesta deberá apoyarse con evidencias adicionales.

La estructura anatómica de la madera de Buddleja sigue el patrón general de la familia en la presencia de elementos de vaso con placas de perforación simple, engrosamientos helicoidales, traqueidas vasculares algunas veces presentes, fibras septadas con punteaduras simples o con areolas reducidas; radios heterogéneos de 1 a 3 series; parénquima axial escaso y sin floema intraxilemático. Sin embargo, se observan algunas diferencias en el número de células de los radios, el tipo de contenidos en vasos y radios y en los datos cuantitativos como son: el diámetro de las punteaduras intervasculares y la altura y anchura de radios. Carlquist (1997) cita que el número de caracteres es tan pequeño que no permite reconocer alguna característica con criterio infragenérico o específico dentro del género. Sin embargo, el análisis de similitud mostró la existencia de dos grupos de especies que deberán confirmarse con caracteres adicionales a los de la madera. Un análisis numérico de los datos cuantitativos podría ayudar a establecer la correspondencia alométrica entre las células de la madera y el hábito de las especies.

\section{RECONOCIMIENTOS}

Agradecemos a los curadores de las xilotecas TWTw, Kw, Uw, Tw, FPRLw, BFHw, CTFw y MEXUw por el envío de las muestras de madera; a la Doctora Eliane Norman, del departamento de Biología de la Universidad de Stetson por la revisión y actualización de los nombres científicos de las especies del género Buddleja; al Sr. José García, curador del herbario del Instituto de Investigaciones Desérticas de la U.A.S.L.P. y al Doctor Mario Ishiki, curador del herbario de la División de la Conservación de la Biodiversidad en el Colegio de la Frontera Sur por su valiosa colaboración en el trabajo de campo realizado en estas zonas; al Biól. José Benitez Flores y a los profesores Alfonso y Araceli Cortés por el apoyo brindado en el trabajo de fotografía y a dos revisores anónimos por sus valiosas sugerencias al manuscrito. Esta investigación se realizó gracias a la beca otorgada por el CONACyT (Reg. No. 118733) al primer autor para la realización de sus estudios de doctorado.

\section{REFERENCIAS}

Aguilar. R.; S., J. Barajas M. y J.D. Tejero D.. 2000. Anatomía de maderas de México: especies de un bosque mesófilo de montaña. Instituto de 
Biología. Publicaciones Especiales 17. Universidad Nacional Autónoma de México. México. 87 p.

Avendaño, G.A. 1996. Estudio de las flavanonas y metabolitos secundarios de Buddleia parviflora. Tesis licenciatura. Facultad de Ciencias. Universidad Nacional Autónoma de México. México. $101 \mathrm{p}$.

Baas, P. 1983. Ecological patterns in xylem anatomy. In: T.J. Givinish, ed. On the economy of plant form and function. Harvard Forest. Cambridge University Press. p: 327-352.

Baas, P. 1999. Review and debate: hardwood fibre pits-again! International Association of Wood Anatomist Journal 20(4): 456-457.

Bentham, G. 1846. Scrophulariaceae. In: A. De Candolle, ed. Prodromus systematis naturalis regni vegetabilis. Vol. 10. Treuttel and Wurtz. Paris.

Berlyn, G.P. y J.P. Miksche. 1976. Botanical microtechnique and cytochemistry. lowa State University Press. lowa. $326 \mathrm{p}$.

Bisset, N.G., T.W.J. Gadella, A.J.M. Leeuwenberger, A.M.W. Mennega y W. Punt. 1980. General discussion of relationships between taxa inside and with taxa outside the family. In: A.J.M. Leeuwenberger, ed. Die natürlichen Pflanzenfamilien. Fam. Loganiaceae. Vol. 28b1. Ducker and Humblot. Berlin. p: 3-7.

Bremer, K., M.W. Chase y P.F. Stevens. 1998. An ordinal classification for the families of flowering plants. Annals of the Missouri Botanical Garden 85(4): 531-553.

Brummitt, R.K. 1992. Vascular plant families and genera. Royal Botanic Gardens. Kew, Gran Bretaña. 804 p.
Carlquist, S. 1982. Wood anatomy of Onagraceae: further species; root anatomy; significance of vestured pit and allied structures in dicotyledons. Annals of the Missouri Botanical Garden 69(4): 755-769.

Carlquist, S. 1988. Comparative wood anatomy. Systematic, ecological, and evolutionary aspects of dicotyledon wood. Springer-Verlag. Berlin. 436 p.

Carlquist, S. 1992. Wood anatomy of sympetalous dicotyledon families: a summary, with comments on systematic relationships and evolution of the woody habit. Annals of the Missouri Botanical Garden 79(2): 303-332.

Carlquist, S. 1997. Wood anatomy of Buddlejaceae. Aliso 15(1): 41-56.

Cronquist, A. 1981. An integrated system of classification of flowering plants. Columbia University Press. New York. 1262 p.

Chalk, L. 1983. The effects of ecological conditions on wood anatomy. In: C.R. Metcalfe y L. Chalk, eds. Anatomy of the dicotyledons. Clarendon Press. Oxford. p: 1-12.

Dahlgren, R. 1975. A system of classification of the angiosperms to be used to demonstrate the distribution of characters. Botanical Noticer 128: 119-147.

Dickison, W.C. 1994. A. re-examination of Sanango racemosum. 2. Vegetative and floral anatomy. Taxon 43(4): 601-618.

Gregory, M. 1994. Bibliography of systematic wood anatomy of dicotyledons. International Association of Wood Anatomist Journal Supplement. $266 \mathrm{p}$. 
Harrar, E.S. 1946. Notes on starch grains in septate fibretracheids. Tropical Woods 85: 1-9.

Herendeen, S.P. y R.B. Miller. 2000. Utility of wood anatomical characters in cladistic analyses. International Association of Wood Anatomist Journal 21(3): 247-276.

Houghton, P.J. 1984. Ethnopharmacology of some Buddleja species. Journal of Ethnopharmacology 11(1): 293-308.

Hunziker, A.T. y E. Di Fulvio. 1958. Observaciones morfológicas sobre Peltanthera (Loganiaceae) con referencia a su posición sistemática. Boletín de la Academia Nacional Ciencia. Córdoba 40: 217-228.

Hutchinson, J. 1973. The families of flowering plants. $3^{\text {a }}$ ed. Clarendon Press. Oxford. $463 \mathrm{p}$.

IAWA Committee. 1989. IAWA list of microscopic features for hardwood identification. International Association of Wood Anatomist Bulletin n.s. 10(3): 219-332.

Jensen, S.R. 1992. Systematic implications of the distribution of iridoids and other chemical compounds in the Loganiaceae and other families of the Asteridae. Annals of the Missouri Botanical Garden 79(2): 284-302.

Johansen, J. 1940. Plant microtechnique. McGraw-Hill Book Company. Nueva York. 523 p.

Kribs, D.A. 1935. Salient lines of specialization in the wood rays of dicotyledons. Botanical Gazette 96(1): 547-557.

Leeuwenberg, A.J.M. 1979. The Loganiaceae of Africa XVIII. Buddleja L. II. Revision of the African and Asiatic species. Meded. Landbou- whogeschool Wageningen 79(6): 1163.

Leeuwenberg, A.J.M. y P.W. Leenhouts. 1980. Taxonomy. In: A.J.M. Leeuwenberger, ed. Die natürlichen pflanzenfamilien. Family Loganiaceae. Vol. 28b1. Ducker and Humblot. Berlin. p: 8-96.

Mabberley, D.J. 1987. The plant-book. Cambridge University Press. Cambridge. $707 p$.

Magendans, J.F.C. 1999. Response to Baas' review of my articles in "morphology of pits in hardwood fibres". International Association of Wood Anatomist Journal 20(4): 458459.

Media Cybernetics. 1997. Image-Pro Plus. Version 3.1 para windows. Maryland. $480 \mathrm{p}$.

Mennega, A.M.W. 1980. Anatomy of the secondary xylem. In: A.J.M. Leeuwenberg, ed. Die natürlichen pflanzenfamilien. Angiospermae: Ordnung Getianales family Loganiaceae. Vol. 28b1. Ducker and Humblot. Berlin. p: 112-161.

Metcalfe, C.R. y L. Chalk. 1950. Anatomy of the dicotyledons. Vol. II. Clarendon Press. Oxford. p: 126-152.

Metcalfe, C.R. y L. Chalk. 1983. Anatomy of dicotyledons, Vol II. 2nd edition. Clarendon Press. Oxford. 297 p.

Norman, M.E. 1966. The genus Buddleia in North America. Gentes Herbarium 10: 47-116.

Ordaz, P.C. 1996. Evaluación in vitro de la actividad amebicida de compuestos obtenidos de Buddleia cordata sobre varias especies de Acanthamoeba, Hartmannella y Vahlkampfia. Tesis licenciatura. Escuela Nacional de Estudios Profesionales Iztacala, Universidad Nacional Autónoma de 
México. Tlalnepantla, Edo. de México. 59 p.

Ortiz Z., E.H. 1996 Actividad antibacterina de la raíz de Buddleia cordata. Tesis licenciatura. Escuela Nacional de Estudios Profesionales Iztacala, Universidad Nacional Autónoma de México. Tlalnepantla, Edo. de México. 75 p.

Oxelman, B., M. Backlund y B. Bremer. 1999. Relationships of the Buddlejaceae s.l. investigated using parsimony jackknife and branch support analysis of chloroplast $n d h F$ and $r b c L$ sequence data. Systematic Botany 24(2): 164-182.

Quintanar, A., C. de la Paz P.O., I. de la Cruz L y D. Razo B. 1996. Anatomía de la madera de ocho especies de angiospermas de clima templado. Boletín de la Sociedad Botánica de México 58: 5-14.

Rajput, S.K. y K.S. Rao. 1999. Nucleated wood fibres in some members of Combretaceae. International Association of Wood Anatomist Journal 20(1): 79-83.

Rohlf, F. J. 1993. NTSYS. Numerical taxonomy and multivariate analysis system. Version 1.80. Nueva York.

Sneath, P.H. A. y R.R. Sokal. 1973. Numerical taxonomy. The principles and practice of numerical classifycation. W.H. Freeman Company. San Francisco, California. 573 p.
Spackman, W. y B.G.L. Swamy. 1949. The nature and occurrence of septate fibres in dicotyledons. American Journal of Botany 36: 804. (Abstract).

Standley, P.C. 1924. Trees and shrubs of Mexico. Contribution to the U.S. National Museum Herbarium 23: 1143-1147.

Solereder, H. 1895. Loganiaceae. In: A. Engler y K. Prantl, eds. Die natürlichen pflanzenfamilien. Vol 4(2): 19-50.

Struwe, L. y V.A. Albert. 1997. Floristics, cladistics, and classification: three case studies in Gentianales. In: J.M. Dransfield, J.E. Coade y D.A. Simpson, eds. Plant diversity in Malesia. Third International Flora Malesiana. Symposium 1995. Flora Malesiana. p: 321-352.

Terrazas, T. y T.L. Wendt. 1995. Systematic wood anatomy of the genus Tapirira Aublet (Anacardiaceae) - A numerical approach. Brittonia 47(2): 109-129.

Wilhelm, K. 1910. Die samenpflansen. Wien, Leipzig, Alemania.

Wolkinger, F. 1970. Morphologie und systematische Verbreitung der lebenden Holzfasern bei Sträuchern und Bäumen. II. Zur histologie. Holzforchung 24: 141-151.»

1 Programa de Botánica. Colegio de Postgraduados. 56230 Montecillo, Estado de México. México.

2 Escuela Nacional de Estudios Profesionales Iztacala UNAM. Biología. Avenida de los Barrios s/n. Los Reyes Iztacala. 54090 Tlalnepantla, Estado de México. México.

Manuscrito recibido el 24 de enero de 2001

Aceptado el 29 de mayo de 2001

Este documento se debe citar como:

Aguilar R., S. y T. Terrazas. 2001. Anatomía de la madera de Buddleja L. (Buddlejaceae): análisis fenético. Madera y Bosques 7(2):63-85 
Apéndice I. Lista de caracteres usados en el análisis fenético de Buddleja

\begin{tabular}{lcc}
\hline \multicolumn{3}{c}{ CARACTERES CUALITATIVOS } \\
\hline \multicolumn{1}{c}{ Vasos } & Presente & Ausente \\
\hline 1. Punteaduras vaso-radio con bordes reducidos & 0 & 1 \\
2. Tílides comunes & 0 & 1 \\
3. Cristales conglomerados & 0 & 1 \\
4. Engrosamientos helicoidales & 0 & 1 \\
5. Engrosamientos helicoidales en todos los vasos & 0 & 1 \\
6. Traqueidas vasculares & 0 & 1
\end{tabular}

Parénquima axial

7. Parénquima axial

8. Apotraqueal difuso

9. Paratraqueal escaso y/o vasicéntrico muy escaso

10. Marginal

11. Gotas esféricas

$0 \quad 1$

$0 \quad 1$

$0 \quad 1$

$0 \quad 1$

$0 \quad 1$

Radios

12. Número de células (series) de 2-4 (angostos)

13. Número de células (series) de 5-7 (2-4) (anchos)

14. Inclusiones, depósitos obscuros

15. Cristales prismáticos pequeños

16. Cristales prismáticos medianos

17. Cristales prismáticos grandes

Fibras

18. Punteaduras semiareoladas

19. Punteaduras simples

20. Septos

21. Inclusiones, depósitos obscuros

22. Anillos de crecimiento

$\begin{array}{ll}0 & 1 \\ 0 & 1 \\ 0 & 1 \\ 0 & 1 \\ 0 & 1 \\ 0 & 1\end{array}$


Apéndice I. Lista de caracteres usados en el análisis fenético de Buddleja (continuación)

\begin{tabular}{|c|c|c|}
\hline \multicolumn{3}{|l|}{ CARACTERES CUANTITATIVOS } \\
\hline Vasos & Presente & Ausente \\
\hline 23. Diámetro de vaso ancho $\leq 33 \mu \mathrm{m}$ & 0 & 1 \\
\hline 24. Diámetro de vaso ancho 34-66 $\mu \mathrm{m}$ & 0 & 1 \\
\hline 25. Diámetro de vaso ancho $\geq 67 \mu \mathrm{m}$ & 0 & 1 \\
\hline 26. Diámetro de vaso angosto $\leq 17 \mu \mathrm{m}$ & 0 & 1 \\
\hline 27. Diámetro de vasos angosto $18-35 \mu \mathrm{m}$ & 0 & 1 \\
\hline 28. Diámetro de vasos angostos $\geq 36 \mu \mathrm{m}$ & 0 & 1 \\
\hline 29. Longitud elemento de vaso ancho $\leq 207 \mu \mathrm{m}$ & 0 & 1 \\
\hline 30. Longitud elemento de vaso ancho $208-413 \mu \mathrm{m}$ & 0 & 1 \\
\hline 31. Longitud elemento de vaso ancho $\geq 414 \mu \mathrm{m}$ & 0 & 1 \\
\hline 32. Longitud elemento de vaso angosto $\leq 500 \mu \mathrm{m}$ & 0 & 1 \\
\hline 33. Longitud elemento de vaso angosto $>500 \mu \mathrm{m}$ & 0 & 1 \\
\hline 34. Diámetro punteadura intervascular $\leq 7.0 \mu \mathrm{m}$ & 0 & 1 \\
\hline 35. Diámetro punteadura intervascular $>8.0 \mu \mathrm{m}$ & 0 & 1 \\
\hline \multicolumn{3}{|l|}{ Fibras } \\
\hline 36. Diámetro total de fibra $\leq 15 \mu \mathrm{m}$ & 0 & 1 \\
\hline 37. Diámetro total de fibra $\geq 16 \mu \mathrm{m}$ & 0 & 1 \\
\hline 38. Longitud de fibra $\leq 384 \mu \mathrm{m}$ & 0 & 1 \\
\hline 39. Longitud de la fibra 385-767 $\mu \mathrm{m}$ & 0 & 1 \\
\hline 40. Longitud de la fibra $\geq 768 \mu \mathrm{m}$ & 0 & 1 \\
\hline 41. Grosor de la pared de la fibra $\leq 3.3 \mu \mathrm{m}$ & 0 & 1 \\
\hline 42. Grosor de la pared de la fibra $\geq 3.4 \mu \mathrm{m}$ & 0 & 1 \\
\hline \multicolumn{3}{|l|}{ Radios } \\
\hline 43. Altura de radio multiseriado $\leq 306 \mu \mathrm{m}$ & 0 & 1 \\
\hline 44. Altura de radio multiseriado $307-612 \mu \mathrm{m}$ & 0 & 1 \\
\hline 45. Altura de radio multiseriado $\geq 613 \mu \mathrm{m}$ & 0 & 1 \\
\hline 46. Ancho de radio multiseriado $\leq 12 \mu \mathrm{m}$ & 0 & 1 \\
\hline 47. Ancho de radio multiseriado $24-47 \mu \mathrm{m}$ & 0 & 1 \\
\hline 48. Ancho de radio multiseriado $\geq 48 \mu \mathrm{m}$ & 0 & 1 \\
\hline
\end{tabular}


Apéndice II. Matriz de similitud producida con el coeficiente de asociación de DiceSorensen para 48 caracteres anatómicos de la madera de 41 especies de Buddleja

\begin{tabular}{|c|c|c|c|c|c|c|c|c|c|c|c|c|c|c|c|c|c|c|c|c|c|}
\hline alternifolia & $\begin{array}{l}\text { alte } \\
1.00\end{array}$ & ame & asi & colv & cor & cris & cur & dav & eleg & glo & inc & long & macr & mad & marr & niti & nive & pani & parv & poly & pulc \\
\hline americana & 0.78 & 1.00 & & & & & & & & & & & & & & & & & & & \\
\hline asiatica & 0.86 & 0.88 & 1.00 & & & & & & & & & & & & & & & & & & \\
\hline colvilei & 0.71 & 0.74 & 0.73 & 1.00 & & & & & & & & & & & & & & & & & \\
\hline cordata & 0.81 & 0.81 & 0.89 & 0.68 & 1.00 & & & & & & & & & & & & & & & & \\
\hline crispa & 0.79 & 0.72 & 0.84 & 0.65 & 0.73 & 1.00 & & & & & & & & & & & & & & & \\
\hline curviflora & 0.86 & 0.74 & 0.85 & 0.67 & 0.86 & 0.81 & 1.00 & & & & & & & & & & & & & & \\
\hline davidii & 0.86 & 0.77 & 0.85 & 0.70 & 0.92 & 0.75 & 0.88 & 1.00 & & & & & & & & & & & & & \\
\hline elegans & 0.79 & 0.76 & 0.81 & 0.57 & 0.82 & 0.76 & 0.86 & 0.84 & 1.00 & & & & & & & & & & & & \\
\hline globosa & 0.86 & 0.80 & 0.88 & 0.64 & 0.92 & 0.78 & 0.91 & 0.94 & 0.86 & 1.00 & & & & & & & & & & & \\
\hline incana & 0.88 & 0.73 & 0.81 & 0.72 & 0.85 & 0.70 & 0.86 & 0.86 & 0.77 & 0.86 & 1.00 & & & & & & & & & & \\
\hline longifolia & 0.77 & 0.88 & 0.85 & 0.70 & 0.86 & 0.68 & 0.76 & 0.82 & 0.84 & 0.82 & 0.72 & 1.00 & & & & & & & & & \\
\hline macrostachya & 0.71 & 0.80 & 0.70 & 0.86 & 0.68 & 0.59 & 0.67 & 0.70 & 0.63 & 0.67 & 0.72 & 0.76 & 1.00 & & & & & & & & \\
\hline madagasc & 0.68 & 0.84 & 0.81 & 0.70 & 0.74 & 0.71 & 0.76 & 0.70 & 0.75 & 0.73 & 0.72 & 0.81 & 0.78 & 1.00 & & & & & & & \\
\hline marrubifolia & 0.69 & 0.69 & 0.74 & 0.62 & 0.78 & 0.69 & 0.77 & 0.77 & 0.85 & 0.80 & 0.67 & 0.77 & 0.62 & 0.65 & 1.00 & & & & & & \\
\hline nítida & 0.69 & 0.81 & 0.77 & 0.71 & 0.72 & 0.66 & 0.68 & 0.68 & 0.67 & 0.71 & 0.67 & 0.80 & 0.80 & 0.88 & 0.57 & 1.00 & & & & & \\
\hline nivea & 0.66 & 0.78 & 0.77 & 0.71 & 0.78 & 0.69 & 0.77 & 0.74 & 0.70 & 0.74 & 0.67 & 0.74 & 0.68 & 0.80 & 0.69 & 0.78 & 1.00 & & & & \\
\hline paniculata & 0.66 & 0.81 & 0.80 & 0.71 & 0.75 & 0.73 & 0.77 & 0.71 & 0.70 & 0.74 & 0.67 & 0.71 & 0.71 & 0.82 & 0.66 & 0.72 & 0.87 & 1.00 & & & \\
\hline parviflora & 0.84 & 0.83 & 0.89 & 0.77 & 0.87 & 0.72 & 0.83 & 0.80 & 0.72 & 0.80 & 0.78 & 0.86 & 0.73 & 0.76 & 0.68 & 0.75 & 0.75 & 0.75 & 1.00 & & \\
\hline polystachya & 0.83 & 0.77 & 0.82 & 0.70 & 0.83 & 0.78 & 0.97 & 0.85 & 0.84 & 0.88 & 0.84 & 0.79 & 0.70 & 0.78 & 0.74 & 0.71 & 0.80 & 0.80 & 0.86 & 1.00 & \\
\hline pulchella & 0.73 & 0.80 & 0.79 & 0.76 & 0.73 & 0.65 & 0.69 & 0.75 & 0.68 & 0.72 & 0.72 & 0.75 & 0.84 & 0.84 & 0.61 & 0.82 & 0.74 & 0.74 & 0.69 & 0.66 & 1.00 \\
\hline saligna & 0.73 & 0.67 & 0.75 & 0.60 & 0.80 & 0.77 & 0.84 & 0.81 & 0.80 & 0.84 & 0.80 & 0.69 & 0.63 & 0.75 & 0.70 & 0.67 & 0.67 & 0.73 & 0.66 & 0.81 & 0.68 \\
\hline salviifolia & 0.88 & 0.76 & 0.84 & 0.66 & 0.88 & 0.80 & 0.98 & 0.89 & 0.88 & 0.92 & 0.88 & 0.78 & 0.69 & 0.75 & 0.79 & 0.67 & 0.76 & 0.76 & 0.81 & 0.95 & 0.68 \\
\hline scordioides & 0.76 & 0.73 & 0.72 & 0.69 & 0.76 & 0.70 & 0.84 & 0.78 & 0.74 & 0.81 & 0.74 & 0.72 & 0.69 & 0.66 & 0.83 & 0.58 & 0.73 & 0.73 & 0.79 & .87 & 0.56 \\
\hline sessiliflora & 0.78 & 0.71 & 0.83 & 0.61 & 0.87 & 0.81 & 0.92 & 0.89 & 0.84 & 0.89 & 0.78 & 0.76 & 0.58 & 0.73 & 0.78 & 0.65 & 0.78 & 0.75 & 0.77 & 0.89 & 0.66 \\
\hline thyrsoides & 0.79 & 0.73 & 0.75 & 0.57 & 0.82 & 0.76 & 0.84 & 0.84 & 0.85 & 0.84 & 0.82 & 0.72 & 0.63 & 0.72 & 0.73 & 0.64 & 0.67 & 0.67 & 0.69 & 81 & 0.65 \\
\hline anchoensis & 0.80 & 0.90 & 0.86 & 0.71 & 0.86 & 0.69 & 0.83 & 0.90 & 1.00 & 0.90 & 0.83 & 0.96 & 0.83 & 0.90 & 0.93 & 0.82 & 0.82 & 0.82 & 0.71 & 0.83 & 0.86 \\
\hline araucana & 0.71 & 0.77 & 0.82 & 0.74 & 0.85 & 0.64 & 0.82 & 0.89 & 0.93 & 0.82 & 0.73 & 0.96 & 0.75 & 0.83 & 0.92 & 0.78 & 0.85 & 0.78 & 0.76 & 0.82 & 0.82 \\
\hline bullata & 0.82 & 0.93 & 0.96 & 0.81 & 0.89 & 0.80 & 0.93 & 0.93 & 0.90 & 0.93 & 0.86 & 0.86 & 0.86 & 1.00 & 0.82 & 0.92 & 0.92 & 0.92 & 0.81 & 0.93 & 0.93 \\
\hline cordobensis & 0.75 & 0.83 & 0.80 & 0.64 & 0.82 & 0.61 & 0.80 & 0.86 & 0.96 & 0.93 & 0.77 & 0.93 & 0.80 & 0.83 & 0.96 & 0.78 & 0.75 & 0.75 & 0.66 & 0.80 & 0.80 \\
\hline domingensis & 0.80 & 0.90 & 0.86 & 0.71 & 0.86 & 0.69 & 0.83 & 0.90 & 1.00 & 0.90 & 0.83 & 0.96 & 0.83 & 0.90 & 0.93 & 0.82 & 0.82 & 0.82 & 0.71 & 0.83 & 0.86 \\
\hline indica & 0.75 & 0.86 & 0.89 & 0.74 & 0.82 & 0.72 & 0.86 & 0.86 & 0.90 & 0.86 & 0.80 & 0.86 & 0.80 & 0.93 & 0.82 & 0.85 & 0.85 & 0.85 & 0.74 & 0.86 & 0.89 \\
\hline mendozensis & 0.71 & 0.77 & 0.75 & 0.66 & 0.85 & 0.56 & 0.75 & 0.89 & 0.86 & 0.89 & 0.73 & 0.89 & 0.75 & 0.77 & 0.92 & 0.71 & 0.78 & 0.71 & 0.69 & 0.75 & 0.75 \\
\hline racemosa & 0.75 & 0.81 & 0.80 & 0.71 & 0.89 & 0.61 & 0.80 & 0.93 & 0.96 & 0.86 & 0.77 & 1.00 & 0.80 & 0.81 & 0.96 & 0.75 & 0.82 & 0.75 & 0.74 & 0.80 & 0.82 \\
\hline rufescens & 0.83 & 0.96 & 0.90 & 0.75 & 0.90 & 0.74 & 0.87 & 0.93 & 0.90 & 0.93 & 0.87 & 0.87 & 0.87 & 0.96 & 0.83 & 0.90 & 0.86 & 0.86 & 0.75 & 0.87 & 0.90 \\
\hline skutchii & 0.82 & 0.93 & 0.96 & 0.81 & 0.89 & 0.80 & 0.93 & 0.93 & 0.90 & 0.93 & 0.86 & 0.86 & 0.86 & 1.00 & 0.82 & 0.92 & 0.92 & 0.92 & 0.81 & 0.93 & 0.96 \\
\hline stachyoides & 0.83 & 0.96 & 0.93 & 0.80 & 0.90 & 0.78 & 0.87 & 0.93 & 0.90 & 0.93 & 0.90 & 0.87 & 0.87 & 0.96 & 0.83 & 0.86 & 0.90 & 0.90 & 0.75 & 0.87 & 0.90 \\
\hline tubiflora & 0.66 & 0.75 & 0.78 & 0.69 & 0.81 & 0.58 & 0.78 & 0.85 & 0.89 & 0.78 & 0.68 & 0.92 & 0.71 & 0.82 & 0.88 & 0.76 & 0.81 & 0.74 & 0.72 & 0.78 & 0.78 \\
\hline tucumanensis & 0.73 & 0.87 & 0.90 & 0.75 & 0.80 & 0.74 & 0.83 & 0.83 & 0.93 & 0.83 & 0.81 & 0.90 & 0.77 & 0.93 & 0.86 & 0.82 & 0.86 & 0.86 & 0.71 & 0.83 & 0.86 \\
\hline utahensis & 0.78 & 0.83 & 0.82 & 0.74 & 0.92 & 0.64 & 0.82 & 0.96 & 0.86 & 0.96 & 0.80 & 0.89 & 0.82 & 0.83 & 0.92 & 0.78 & 0.85 & 0.78 & 0.76 & 0.82 & 0.85 \\
\hline \multirow[t]{2}{*}{ australis } & 0.72 & 0.81 & 0.76 & 0.64 & 0.84 & 0.60 & 0.76 & 0.88 & 0.96 & 0.84 & 0.74 & 0.96 & 0.76 & 0.81 & 0.92 & 0.75 & 0.76 & 0.72 & 0.66 & 0.76 & 0.80 \\
\hline & alte & ame & asi & colv & cor & cris & cur & dav & eleg & glo & inc & long & macr & mad & marr & niti & nive & pani & parv & poly & pulc \\
\hline
\end{tabular}


Apéndice II. Matriz de similitud producida con el coeficiente de asociación de DiceSorensen para 48 caracteres anatómicos de la madera de 41 especies de Buddleja (cont.)

\begin{tabular}{|c|c|c|c|c|c|c|c|c|c|c|c|c|c|c|c|c|c|c|c|c|}
\hline alternifolia & sali & salv & scor & sess & thyr & anch & arau & bull & cord & domi & indi & mend & race & rufr & skut & stac & tubi & tucu & utah & aust \\
\hline americana & & & & & & & & & & & & & & & & & & & & \\
\hline asiatica & & & & & & & & & & & & & & & & & & & & \\
\hline colvilei & & & & & & & & & & & & & & & & & & & & \\
\hline cordata & & & & & & & & & & & & & & & & & & & & \\
\hline crispa & & & & & & & & & & & & & & & & & & & & \\
\hline curviflora & & & & & & & & & & & & & & & & & & & & \\
\hline davidii & & & & & & & & & & & & & & & & & & & & \\
\hline elegans & & & & & & & & & & & & & & & & & & & & \\
\hline globosa & & & & & & & & & & & & & & & & & & & & \\
\hline incana & & & & & & & & & & & & & & & & & & & & \\
\hline longifolia & & & & & & & & & & & & & & & & & & & & \\
\hline macrostachya & & & & & & & & & & & & & & & & & & & & \\
\hline madagasc & & & & & & & & & & & & & & & & & & & & \\
\hline marrubifolia & & & & & & & & & & & & & & & & & & & & \\
\hline nítida & & & & & & & & & & & & & & & & & & & & \\
\hline nivea & & & & & & & & & & & & & & & & & & & & \\
\hline paniculata & & & & & & & & & & & & & & & & & & & & \\
\hline parviflora & & & & & & & & & & & & & & & & & & & & \\
\hline polystachya & & & & & & & & & & & & & & & & & & & & \\
\hline pulchella & & & & & & & & & & & & & & & & & & & & \\
\hline saligna & 1.00 & & & & & & & & & & & & & & & & & & & \\
\hline salviifolia & 0.86 & 1.00 & & & & & & & & & & & & & & & & & & \\
\hline scordioides & 0.71 & 0.86 & 1.00 & & & & & & & & & & & & & & & & & \\
\hline sessiliflora & 0.88 & 0.90 & 0.79 & 1.00 & & & & & & & & & & & & & & & & \\
\hline thyrsoides & 0.77 & 0.85 & 0.74 & 0.81 & 1.00 & & & & & & & & & & & & & & & \\
\hline anchoensis & 0.78 & 0.87 & 0.75 & 0.78 & 0.90 & 1.00 & & & & & & & & & & & & & & \\
\hline araucana & 0.69 & 0.80 & 0.74 & 0.84 & 0.80 & 0.93 & 1.00 & & & & & & & & & & & & & \\
\hline bullata & 0.81 & 0.90 & 0.78 & 0.88 & 0.93 & 0.90 & 0.89 & 1.00 & & & & & & & & & & & & \\
\hline cordobensis & 0.74 & 0.83 & 0.78 & 0.74 & 0.86 & 0.96 & 0.89 & 0.86 & 1.00 & & & & & & & & & & & \\
\hline domingensis & 0.78 & 0.87 & 0.75 & 0.78 & 0.90 & 1.00 & 0.93 & 0.90 & 0.96 & 1.00 & & & & & & & & & & \\
\hline indica & 0.74 & 0.83 & 0.71 & 0.81 & 0.86 & 0.90 & 0.89 & 0.93 & 0.86 & 0.90 & 1.00 & & & & & & & & & \\
\hline mendozensis & 0.69 & 0.80 & 0.81 & 0.76 & 0.80 & 0.86 & 0.85 & 0.82 & 0.89 & 0.86 & 0.89 & 1.00 & & & & & & & & \\
\hline racemosa & 0.74 & 0.83 & 0.78 & 0.81 & 0.83 & 0.96 & 0.96 & 0.86 & 0.93 & 0.96 & 0.86 & 0.89 & 1.00 & & & & & & & \\
\hline rufescens & 0.82 & 0.90 & 0.80 & 0.82 & 0.96 & 0.93 & 0.83 & 0.96 & 0.90 & 0.93 & 0.90 & 0.83 & 0.87 & 1.00 & & & & & & \\
\hline skutchii & 0.81 & 0.90 & 0.78 & 0.88 & 0.93 & 0.90 & 0.89 & 1.00 & 0.86 & 0.90 & 0.93 & 0.82 & 0.86 & 0.96 & 1.00 & & & & & \\
\hline stachyoides & 0.82 & 0.90 & 0.80 & 0.82 & 0.93 & 0.93 & 0.83 & 0.96 & 0.87 & 0.93 & 0.90 & 0.83 & 0.87 & 1.00 & 0.96 & 1.00 & & & & \\
\hline tubiflora & 0.72 & 0.75 & 0.69 & 0.88 & 0.78 & 0.89 & 0.96 & 0.85 & 0.85 & 0.89 & 0.85 & 0.81 & 0.92 & 0.82 & 0.85 & 0.80 & 1.00 & & & \\
\hline tucumanensis & 0.71 & 0.81 & 0.68 & 0.78 & 0.83 & 0.96 & 0.93 & 0.93 & 0.90 & 0.96 & 0.93 & 0.80 & 0.90 & 0.90 & 0.93 & 0.90 & 0.89 & 1.00 & & \\
\hline utahensis & 0.76 & 0.86 & 0.88 & 0.84 & 0.86 & 0.86 & 0.85 & 0.89 & 0.89 & 0.86 & 0.82 & 0.92 & 0.89 & 0.90 & 0.89 & 0.90 & 0.81 & 0.80 & 1.00 & \\
\hline australis & 0.75 & 0.81 & 0.72 & 0.80 & 0.84 & 0.96 & 0.92 & 0.84 & 0.92 & 0.96 & 0.84 & 0.84 & 0.96 & 0.88 & 0.84 & 0.85 & 0.88 & 0.88 & 0.84 & 1.00 \\
\hline & sali & salv & scor & sess & thyr & anch & arau & bull & cord & domi & indi & mend & race & rufr & skut & stac & tubi & tucu & utah & aust \\
\hline
\end{tabular}

